\title{
THERMAL STRESSES IN CHIRAL PLATES
}

\section{Ieşan ${ }^{1}$ and R. Quintanilla ${ }^{2}$}

${ }^{1}$ Department of Mathematics, Al.I. Cuza University and Octav Mayer Institute of Mathematics (Romanian Academy), Bd. Carol I, nr. 8, 700508 Iaşi, Romania

${ }^{2}$ Department of Mathematics, ESEIAAT, Polytechnic University of Catalonia, Colón, 11, 08222 Terrassa, Barcelona, Spain

\section{Acknowledgments}

R.Q. is supported by the Project "Análisis Matemático de las Ecuaciones en Derivada Parciales de la Termomecánica“ (MTM2013-42004-P) of the Spanish Ministry of Economy and Competitiveness. At the same time this work is part of the Project "Análisis Matemático de Problemas de la Termomecánica“(MTM2016-74934-P) which is currently submitted to the Spanish Ministry of Economy and Competitiveness. 


\section{THERMAL STRESSES IN CHIRAL PLATES}

This paper is concerned with the linear theory of chiral Cosserat thermoelastic bodies. We investigate the deformation of chiral plates. First, we present the basic equations which govern the deformation of thin plates. Then, we present reciprocity and uniqueness results. In the next section we establish the instability of solutions whenever the internal energy is negative. We use a semigroup approach to prove the existence of solution. The deformation of an infinite plate with a circular hole is investigated.

Keywords: Thermal stresses in plates; Chiral materials; Uniqueness results.

\section{INTRODUCTION}

The behavior of chiral materials is of interest for the investigation of carbon nanotubes, auxetic materials, bones, honeycomb structures, as well as composites with inclusions. The deformation of chiral elastic materials cannot be described within clas-

sical elasticity $[1,2]$. Various authors have studied the behavior of chiral materials by using the theory of Cosserat elasticity (see, e.g., [3-10] and references therein). The Cosserat theory studies continua with oriented particles, in which each material point has the six degrees of freedom of a rigid body. The basic equations of the linear theory of Cosserat thermoelasticity have been studied in various books (see, e.g., Nowacki [11], Eringen [12], Dyszlewicz [13]). The deformation of achiral Cosserat elastic plates has been investigated by various authors. A detailed analysis of the results established in 
this theory have been presented in [14].

In this paper we use the results of Eringen [15], Nowacki [16] and Inan[17] to derive a theory of isotropic chiral plates. We assume that on the upper and lower faces of the plate there are prescribed the surface traction, the surface moment and the heat flux. We show that, in contrast with the theory of achiral plates, the stretching and flexure cannot be treated independently of each other. The paper is structured as follows. First, we present the basic equations of homogenenous and isotropic chiral Cosserat thermoelastic solids. Then, we establish a theory of thermoelastic thin plates. In the following section we present reciprocity and uniqueness results in the dynamic theory. Then, by means of the logarithmic convexity arguments we investigate the instability of solutions. We use a semigroup approach to establish an existence result. Finally, the deformation of an infinite plate with a circular hole is investigated. It is shown that a constant thermal field in a chiral plate produces a bending effect.

\section{BASIC EQUATIONS}

In this section we present the basic equations of the thermoelasticity for isotropic chiral Cosserat continua. We refer the motion of the continuum to a fixed system of rectangular Cartesian axes $O x_{k},(k=1,2,3)$. We consider a body that at time $t_{0}$ occupies the regular region $B$ of Euclidean three-dimensional space and is bounded by the surface $\partial B$. We designate by $\boldsymbol{n}$ the outward unit normal of $\partial B$. Letters in boldface stand for tensors of an order $p \geq 1$, and if $\boldsymbol{v}$ has the order $p$, we write $v_{i j \ldots s}$ ( $p$ subscripts) for the components of $\boldsymbol{v}$ in the Cartesian coordinate system. We shall employ the usual 
summation and differentiation conventions: Latin subscripts are understood to range over the integers $(1,2,3)$ whereas Greek subscripts to the range $(1,2)$; summation over repeated subscripts is implied, and subscripts preceded by a comma denote partial differentiation with respect to the corresponding Cartesian coordinate. In all that follows, we use a superposed dot to denote partial differentiation with respect to the time.

We assume that $B$ is occupied by a homogeneous and isotropic chiral Cosserat thermoelastic material. We denote by $u_{i}$ the components of the displacement vector and by $\varphi_{i}$ the components of the microrotation vector. The strain measures in the linear theory are defined by

$$
e_{i j}=u_{j, i}+\varepsilon_{j i k} \varphi_{k} \quad \kappa_{i j}=\varphi_{j, i}
$$

where $\varepsilon_{i j k}$ is the alternating symbol. Let $t_{i j}$ be the stress tensor and let $m_{i j}$ be the couple stress tensor over $B$. The equations of motion of a Cosserat continua can be expressed as

$$
\begin{aligned}
& t_{j i, j}+f_{i}=\rho \ddot{u}_{i} \\
& m_{j i, j}+\varepsilon_{i j k} t_{j k}+g_{i}=J \ddot{\varphi}_{i}
\end{aligned}
$$

where $f_{i}$ is the body force, $g_{i}$ is the body couple, $\rho$ is the reference mass density, and $J$ is a coefficient of inertia. The energy equation is given by

$$
\rho T_{0} \dot{\eta}=q_{i, i}+s
$$

where $\eta$ is the entropy, $q_{i}$ is the heat flux vector, $s$ is the heat supply, and $T_{0}$ is the constant absolute temperature of the body in the reference configuration. 
In the context of the linear theory of isotropic chiral and homogeneous thermoelastic bodies the constitutive equations have the form $[1,12,13]$

$$
\begin{aligned}
& t_{i j}=\lambda e_{r r} \delta_{i j}+(\mu+\kappa) e_{i j}+\mu e_{j i}+C_{1} \kappa_{s s} \delta_{i j}+C_{2} \kappa_{j i}+C_{3} \kappa_{i j}-b_{1} \theta \delta_{i j} \\
& m_{i j}=\alpha \kappa_{r r} \delta_{i j}+\beta \kappa_{j i}+\gamma \kappa_{i j}+C_{1} e_{r r} \delta_{i j}+C_{2} e_{j i}+C_{3} e_{i j}-b_{2} \theta \delta_{i j} \\
& \rho \eta=b_{1} e_{r r}+b_{2} \kappa_{r r}+a \theta \quad q_{i}=k \theta_{, i}
\end{aligned}
$$

where $\delta_{i j}$ is the Kronecker delta, $\theta$ is the temperature measured from the constant absolute temperature of the reference state, and $\lambda, \mu, \kappa, \alpha, \beta, \gamma, C_{1}, C_{2}, C_{3}, b_{1}, b_{2}, a$ and $k$ are constitutive constants. In the case of an achiral material the coefficients $C_{1}, C_{2}, C_{3}$ and $b_{2}$ are equal to zero.

The components of surface traction, the components of surface couple and the heat flux at regular points of $\partial B$ are defined by

$$
t_{i}=t_{j i} n_{j} \quad m_{i}=m_{j i} n_{j} \quad q=q_{i} n_{i}
$$

respectively. Let $P=\left(u_{i}, \varphi_{i}, \theta\right)$. We say that $P$ is an admissible process on $B \times \mathcal{T}$ provided: (i) $u_{i}$ and $\varphi_{i}$ are of class $C^{2}$ on $B \times \mathcal{T}$; (ii) $\theta$ is of class $C^{2,1}$ on $B \times \mathcal{T}$; (iii) $u_{i}, \varphi_{i}$ and $\theta$ are of class $C^{1}$ on $\bar{B} \times \mathcal{T}$. We assume that $f_{i}, g_{i}$ and $s$ are continuous on $B \times \mathcal{T}$ and that $\rho$ and $J$ are positive constants.

\section{THERMOELASTIC PLATES}

In what follows we assume that the region $B$ refers to the interior of a right cylinder of length $2 h$ with open cross-section $\Sigma$ and the smooth lateral boundary $\Pi$. Let $\Gamma$ be 
the boundary of $\Sigma$. The rectangular Cartesian coordinate frame consists of the origin $O$ and the orthonormal basis $\left\{\boldsymbol{e}_{1}, \boldsymbol{e}_{2}, \boldsymbol{e}_{3}\right\}$. The coordinate frame is supposed to be chosen in such a way that the plane $x_{1} O x_{2}$ is middle plane. Thus, we have

$$
B=\left\{x:\left(x_{1}, x_{2}\right) \in \Sigma,-h<x_{3}<h\right\}, \Pi=\left\{x:\left(x_{1}, x_{2}\right) \in \Gamma,-h<x_{3}<h\right\}
$$

We derive a theory of thin plates of uniform thickness where the displacements, the microrotations and the temperature have the form

$$
\begin{aligned}
& u_{\alpha}=w_{\alpha}\left(x_{1}, x_{2}, t\right)+x_{3} v_{\alpha}\left(x_{1}, x_{2}, t\right) u_{3}=w_{3}\left(x_{1}, x_{2}, t\right) \\
& \varphi_{j}=\Phi_{j}\left(x_{1}, x_{2}, t\right) \\
& \theta=T_{1}\left(x_{1}, x_{2}, t\right)+x_{3} T_{2}\left(x_{1}, x_{2}, t\right) \quad\left(x_{1}, x_{2}, x_{3}\right) \in B t \in \mathcal{T}
\end{aligned}
$$

Following [15-17], to establish a plate theory we perform the following integrations: (i) we integrate equations of balance of momenta with respect to $x_{3}$ over the thickness of the plate; (ii) we take the cross product of the equations of the balance of linear momentum with $x_{3} \boldsymbol{e}_{3}$ and integrate over the thickness of the plate; (iii) we integrate the equation of energy over $x_{3}$ between the limits $-h$ and $h$; (iv) we multiply the equation of energy by $x_{3}$ and integrate over the thickness of the plate. The results of (i) are

$$
\tau_{\beta k, \beta}+F_{k}=\rho \ddot{w}_{k} \quad \mu_{\beta \alpha, \beta}+\varepsilon_{3 \rho \alpha}\left(\tau_{3 \rho}-\tau_{\rho 3}\right)+G_{\alpha}=J \ddot{\Phi}_{\alpha} \quad \mu_{\alpha 3, \alpha}+\varepsilon_{3 \rho \alpha} \tau_{\rho \alpha}+G_{3}=J \ddot{\Phi}_{3}
$$

where

$$
\begin{aligned}
\tau_{i j} & =\frac{1}{2 h} \int_{-h}^{h} t_{i j} d x_{3} \mu_{i j}=\frac{1}{2 h} \int_{-h}^{h} m_{i j} d x_{3} \\
F_{i} & =\frac{1}{2 h} \int_{-h}^{h} f_{i} d x_{3}+\frac{1}{2 h}\left[t_{3 i}\right]_{-h}^{h} G_{i}=\frac{1}{2 h} \int_{-h}^{h} g_{i} d x_{3}+\frac{1}{2 h}\left[m_{3 i}\right]_{-h}^{h}
\end{aligned}
$$


We assume that the functions $t_{i}, m_{i}$ and $q$ are prescribed on the surfaces $x_{3}= \pm h$.

To the equations (6) we add the result of (ii), i.e.,

$$
M_{\beta \alpha, \beta}-2 h \tau_{3 \alpha}+H_{\alpha}=\rho I \ddot{v}_{\alpha}
$$

where we have used the notations

$$
M_{\alpha \beta}=\int_{-h}^{h} x_{3} t_{\alpha \beta} d x_{3} \quad I=\frac{2}{3} h^{3} H_{\alpha}=\int_{-h}^{h} x_{3} f_{\alpha} d x_{3}+\left[x_{3} t_{3 \alpha}\right]_{-h}^{h}
$$

If we integrate the equation (3) with respect to $x_{3}$ between the limits $-h$ and $h$, then we obtain the following equation

$$
\rho T_{0} \dot{\zeta}=\chi_{\alpha, \alpha}+S_{1}
$$

where the functions $\zeta, \chi_{j}$ and $S_{1}$ are defined by

$$
\begin{aligned}
& \zeta=\frac{1}{2 h} \int_{-h}^{h} \eta d x_{3} \chi_{j}=\frac{1}{2 h} \int_{-h}^{h} q_{j} d x_{3} \\
& S_{1}=\frac{1}{2 h} \int_{-h}^{h} s d x_{3}+\frac{1}{2 h}\left[q_{3}\right]_{-h}^{h}
\end{aligned}
$$

The equation which results from the multiplication of Eq. (3) by $x_{3}$ and integration over $x_{3}$ from $x_{3}=-h$ to $x_{3}=h$ can be written in the form

$$
\rho T_{0} \dot{\sigma}=Q_{\alpha, \alpha}-2 h \chi_{3}+S_{2}
$$

where we have used the notations

$$
\sigma=\int_{-h}^{h} x_{3} \eta d x_{3} Q_{\alpha}=\int_{-h}^{h} x_{3} q_{\alpha} d x_{3} S_{2}=\int_{-h}^{h} x_{3} s d x_{3}+\left[x_{3} q_{3}\right]_{-h}^{h}
$$


The functions $F_{j}, G_{j}, H_{\alpha}$ and $S_{\alpha}$ are prescribed. From (1) and (6) we obtain

$$
e_{\alpha \beta}=\gamma_{\alpha \beta}+x_{3} \xi_{\alpha \beta} \quad e_{33}=0 e_{\alpha 3}=\gamma_{\alpha 3} e_{3 \alpha}=\gamma_{3 \alpha} \kappa_{\alpha j}=\eta_{\alpha j} \kappa_{3 j}=0
$$

where

$$
\gamma_{\alpha j}=w_{j, \alpha}+\varepsilon_{j \alpha k} \Phi_{k} \gamma_{3 \alpha}=v_{\alpha}+\varepsilon_{3 \beta \alpha} \Phi_{\beta} \eta_{\alpha k}=\Phi_{k, \alpha} \xi_{\alpha \beta}=v_{\beta, \alpha}
$$

It follows from $(4),(8),(10),(12),(14),(15)$ and $(6)$ that

$$
\begin{aligned}
\tau_{\alpha \beta} & =\lambda \gamma_{\rho \rho} \delta_{\alpha \beta}+(\mu+\kappa) \gamma_{\alpha \beta}+\mu \gamma_{\beta \alpha}+C_{1} \eta_{\rho \rho} \delta_{\alpha \beta} \\
& +C_{2} \eta_{\beta \alpha}+C_{3} \eta_{\alpha \beta}-b_{1} T_{1} \delta_{\alpha \beta} \\
\tau_{\alpha 3} & =(\mu+\kappa) \gamma_{\alpha 3}+\mu \gamma_{3 \alpha}+C_{3} \eta_{\alpha 3} \\
\tau_{3 \alpha} & =(\mu+\kappa) \gamma_{3 \alpha}+\mu \gamma_{\alpha 3}+C_{2} \eta_{\alpha 3} \\
\mu_{\nu \kappa} & =\alpha \eta_{\rho \rho} \delta_{\nu \kappa}+\beta \eta_{\kappa \nu}+\gamma \eta_{\nu \kappa}+C_{1} \gamma_{\rho \rho} \delta_{\nu \kappa}+C_{2} \gamma_{\kappa \nu}+C_{3} \gamma_{\nu \kappa}-b_{2} T_{1} \delta_{\nu \kappa} \\
\mu_{\alpha 3} & =\beta \eta_{3 \alpha}+\gamma \eta_{\alpha 3}+C_{2} \gamma_{3 \alpha}+C_{3} \gamma_{\alpha 3} \\
M_{\alpha \beta} & =I\left[\lambda \xi_{\rho \rho} \delta_{\alpha \beta}+(\mu+\kappa) \xi_{\alpha \beta}+\mu \xi_{\beta \alpha}-b_{1} T_{2} \delta_{\alpha \beta}\right] \\
\rho \zeta & =b_{1} \gamma_{\rho \rho}+b_{2} \eta_{\rho \rho}+a T_{1} \chi_{\alpha}=k T_{1, \alpha} \chi_{3}=k T_{2} \\
\rho \sigma & =I\left(b_{1} \xi_{\rho \rho}+a T_{2}\right) Q_{\alpha}=k I T_{2, \alpha}
\end{aligned}
$$

The basic equations of the theory of chiral plates consist of the equations of motion (7) and (9), the equations of the energy (11) and (13), the constitutive equations (17) and the geometrical equations (16). The field equations can be expressed in terms of the 
functions $w_{k}, \Phi_{k}, v_{\alpha}, T_{1}$ and $T_{2}$. We obtain the following equations

$$
\begin{aligned}
& (\mu+\kappa) \Delta w_{\alpha}+(\lambda+\mu) w_{\rho, \rho \alpha}+C_{3} \Delta \Phi_{\alpha}+\left(C_{1}+C_{2}\right) \Phi_{\rho, \rho \alpha} \\
& +\kappa \varepsilon_{3 \alpha \beta} \Phi_{3, \beta}-b_{1} T_{1, \alpha}+F_{\alpha}=\rho \ddot{w}_{\alpha} \\
& (\mu+\kappa) \Delta w_{3}+C_{3} \Delta \Phi_{3}+\kappa \varepsilon_{3 \alpha \beta} \Phi_{\beta, \alpha}+\mu v_{\rho, \rho}+F_{3}=\rho \ddot{w}_{3} \\
& C_{3} \Delta w_{\alpha}+\left(C_{1}+C_{2}\right) w_{\rho, \rho \alpha}+\gamma \Delta \Phi_{\alpha}+(\alpha+\beta) \Phi_{\rho, \rho \alpha}+\kappa \varepsilon_{3 \alpha \rho}\left(w_{3, \rho}-v_{\rho}\right) \\
& +2\left(C_{3}-C_{2}\right) \varepsilon_{3 \alpha \beta} \Phi_{3, \beta}-2 \kappa \Phi_{\alpha}-b_{2} T_{1, \alpha}+G_{\alpha}=J \ddot{\Phi}_{\alpha} \\
& C_{3} \Delta w_{3}+\gamma \Delta \Phi_{3}+\kappa \varepsilon_{3 \rho \alpha} w_{\alpha, \rho}+2 \varepsilon_{3 \alpha \beta}\left(C_{3}-C_{2}\right) \Phi_{\beta, \alpha} \\
& +C_{2} v_{\alpha, \alpha}-2 \kappa \Phi_{3}+G_{3}=J \ddot{\Phi}_{3} \\
& I\left[(\mu+\kappa) \Delta v_{\alpha}+(\lambda+\mu) v_{\rho, \rho \alpha}-b_{1} T_{2, \alpha}\right] \\
& -2 h\left[\mu w_{3, \alpha}+C_{2} \Phi_{3, \alpha}+\kappa \varepsilon_{3 \beta \alpha} \Phi_{\beta}+(\mu+\kappa) v_{\alpha}\right]+H_{\alpha}=\rho I \ddot{v}_{\alpha} \\
& k \Delta T_{1}-c \dot{T}_{1}-T_{0} b_{1} \dot{w}_{\alpha, \alpha}-T_{0} b_{2} \dot{\Phi}_{\alpha, \alpha}=-S_{1} \\
& I\left[k \Delta T_{2}-c \dot{T}_{2}-T_{0} b_{1} \dot{v}_{\alpha, \alpha}\right]-2 h k T_{2}=-S_{2}
\end{aligned}
$$

where $\Delta$ is the two-dimensional Laplacian, and we have used the notation $c=a T_{0}$. We note that in the case of the centrosymmetric solids the coefficients $C_{k}$ and $b_{2}$ are equal to zero. In this case the system (18) reduces to two uncoupled systems: one for the functions $w_{\alpha}, \Phi_{3}$ and $T_{1}$, and the other for the functions $w_{3}, v_{\alpha}, \Phi_{\alpha}$ and $T_{2}$.

To the field equations we must adjoin initial conditions and boundary conditions. 
The initial conditions are

$$
\begin{aligned}
& w_{j}\left(x_{1}, x_{2}, 0\right)=w_{j}^{0}\left(x_{1}, x_{2}\right) \Phi_{j}\left(x_{1}, x_{2}, 0\right)=\Phi_{j}^{0}\left(x_{1}, x_{2}\right) \\
& v_{\alpha}\left(x_{1}, x_{2}, 0\right)=v_{\alpha}^{0}\left(x_{1}, x_{2}\right) T_{\alpha}\left(x_{1}, x_{2}, 0\right)=T_{\alpha}^{0}\left(x_{1}, x_{2}\right) \\
& \dot{w}_{j}\left(x_{1}, x_{2}, 0\right)=\vartheta_{j}^{0}\left(x_{1}, x_{2}\right) \dot{\Phi}_{j}\left(x_{1}, x_{2}, 0\right)=\omega_{j}^{0}\left(x_{1}, x_{2}\right) \\
& \dot{v}_{\alpha}\left(x_{1}, x_{2}, 0\right)=\eta_{\alpha}^{0}\left(x_{1}, x_{2}\right) \quad\left(x_{1}, x_{2}\right) \in \Sigma
\end{aligned}
$$

where the functions $w_{j}^{0}, \Phi_{j}^{0}, v_{\alpha}^{0}, T_{\alpha}^{0}, \vartheta_{j}^{0}, \omega_{j}^{0}$ and $\eta_{\alpha}^{0}$ are given. The Neumann problem is characterized by the following boundary conditions

$$
\begin{aligned}
& \tau_{\beta j} n_{\beta}=\widetilde{\tau}_{j} \quad \mu_{\beta j} n_{\beta}=\widetilde{\mu}_{j} \quad M_{\beta \alpha} n_{\beta}=\widetilde{M}_{\alpha} \\
& \chi_{\alpha} n_{\alpha}=\widetilde{\chi} \quad Q_{\alpha} n_{\alpha}=\widetilde{Q} \text { on } \Gamma \times \mathcal{T}
\end{aligned}
$$

where the functions $\widetilde{\tau}_{j}, \widetilde{\mu}_{j}, \widetilde{M}_{\alpha}, \widetilde{\chi}$ and $\widetilde{Q}$ are prescribed. In the case of Dirichlet problem the boundary conditions are

$$
w_{j}=\widetilde{w}_{j} \Phi_{j}=\widetilde{\Phi}_{j} v_{\alpha}=\widetilde{v}_{\alpha} T_{\alpha}=\widetilde{T}_{\alpha} \quad \text { on } \Gamma \times \mathcal{T}
$$

where the functions $\widetilde{w}_{j}, \widetilde{\Phi}_{j}, \widetilde{v}_{\alpha}$ and $\widetilde{T}_{\alpha}$ are given.

\section{RECIPROCITY AND UNIQUENESS RESULTS}

In this section we present reciprocity and uniqueness theorems in the framework of the dynamic theory. Let $F$ and $G$ be scalar fields on $\Sigma \times \mathcal{T}$ that are continuous in time. We denote by $F * G$ the convolution of $F$ and $G$, i.e.

$$
F * G(x, t)=\int_{0}^{t} F(x, t-\tau) G(x, \tau) d \tau x \in \Sigma t \in \mathcal{T}
$$


In what follows we suppose that $\mathcal{T}=(0, \infty)$. Let $f$ and $g$ be functions on $\mathcal{T}$ defined by

$$
f(t)=1 g(t)=t \quad t \in \mathcal{T}
$$

If $F$ is a continuous function on $\Sigma \times \mathcal{T}$, then we write $\widehat{F}$ for $f * F$, that is

$$
\widehat{F}(x, t)=\int_{0}^{t} F(x, \tau) d \tau x \in \Sigma t \in \mathcal{T}
$$

We define the functions $W_{1}$ and $W_{2}$ on $\Sigma \times \mathcal{T}$ by

$$
W_{1}=\widehat{S}_{1}+\rho T_{0} \zeta^{0} W_{2}=\widehat{S}_{2}+\rho T_{0} \sigma^{0}
$$

where

$$
\rho \zeta^{0}=b_{1} w_{\alpha, \alpha}^{0}+b_{2} \Phi_{\alpha, \alpha}^{0}+a T_{1}^{0} \rho \sigma^{0}=I\left(b_{1} v_{\alpha, \alpha}^{0}+a T_{2}^{0}\right)
$$

In view of (11), (13), (17), (24) and (25) we obtain

Lemma 1. The functions $\zeta, \sigma \in C^{0,1}, \chi_{\alpha}, Q_{\alpha} \in C^{1,0}$ and $\chi_{3} \in C^{0,0}$ satisfy Eqs. and (13), and the initial conditions $\zeta(x, 0)=\zeta^{0}(x), \sigma(x, 0)=\sigma^{0}(x), x \in \Sigma$, if and only if

$$
\rho T_{0} \zeta=\widehat{\chi}_{\alpha, \alpha}+W_{1} \rho T_{0} \sigma=\widehat{Q}_{\alpha, \alpha}-2 h \widehat{\chi}_{3}+W_{2} \text { on } \Sigma \times[0, \infty)
$$

The proof is immediate.

We consider two data systems of loading for the Neumann problem

$$
\begin{aligned}
& \mathcal{L}^{(\alpha)}=\left\{F_{k}^{(\alpha)}, G_{k}^{(\alpha)}, H_{\beta}^{(\alpha)}, S_{\beta}^{(\alpha)}, w_{k}^{0(\alpha)}, \Phi_{k}^{0(\alpha)}, T_{\beta}^{0(\alpha)}, v_{\rho}^{0(\alpha)}\right. \\
& \left.\vartheta_{j}^{0(\alpha)}, \omega_{j}^{0(\alpha)}, \eta_{\beta}^{0(\alpha)}, \widetilde{\tau}_{j}^{(\alpha)}, \widetilde{\mu}_{j}^{(\alpha)}, \widetilde{M}_{\rho}^{(\alpha)}, \widetilde{\chi}^{(\alpha)}, \widetilde{Q}^{(\alpha)}\right\}(\alpha=1,2)
\end{aligned}
$$


and denote by $A^{(\alpha)}=\left\{w_{k}^{(\alpha)}, \Phi_{k}^{(\alpha)}, v_{\beta}^{(\alpha)}, T_{\beta}^{(\alpha)}, \gamma_{i j}^{(\alpha)}, \eta_{\beta k}^{(\alpha)}, \xi_{\beta \alpha}^{(\alpha)}, \zeta^{(\alpha)}, \sigma^{(\alpha)}, \tau_{i j}^{(\alpha)}, \mu_{\beta j}^{(\alpha)}, M_{\kappa \nu}^{(\alpha)}\right.$, $\left.\chi_{k}^{(\alpha)}, Q_{\beta}^{(\alpha)}\right\}$, a solution corresponding to $\mathcal{L}^{(\alpha)}$. We define the following functions corresponding to the solution $A^{(\alpha)}$,

$$
\begin{aligned}
& \tau_{k}^{(\alpha)}=\tau_{\beta k}^{(\alpha)} n_{\beta} \mu_{k}^{(\alpha)}=\mu_{\beta k}^{(\alpha)} n_{\beta} M_{\rho}^{(\alpha)}=M_{\beta \rho}^{(\alpha)} n_{\beta} \\
& \chi^{(\alpha)}=\chi_{\rho}^{(\alpha)} n_{\rho} Q^{(\alpha)}=Q_{\rho}^{(\alpha)} n_{\rho} \\
& W_{1}^{(\alpha)}=\widehat{S}_{1}^{(\alpha)}+\rho T_{0} \zeta^{0(\alpha)} W_{2}^{(\alpha)}=\widehat{S}_{2}^{(\alpha)}+\rho T_{0} \sigma^{0(\alpha)}
\end{aligned}
$$

We introduce the notations

$$
\begin{aligned}
\Pi_{\kappa \nu}(r, s) & =\int_{\Gamma}\left\{2 h\left[\tau_{j}^{(\kappa)}(r) w_{j}^{(\nu)}(s)+\mu_{j}^{(\kappa)}(r) \Phi_{j}^{(\nu)}(s)-\frac{1}{T_{0}} \widehat{\chi}^{(\kappa)}(r) T_{1}^{(\nu)}(s)\right]\right. \\
& \left.+M_{\beta}^{(\kappa)}(r) v_{\beta}^{(\nu)}(s)-\frac{1}{T_{0}} \widehat{Q}^{(\kappa)}(r) T_{2}^{(\nu)}(s)\right\} d l \\
& +\int_{\Sigma}\left\{2 h\left[F_{j}^{(\kappa)}(r) w_{j}^{(\nu)}(s)+G_{j}^{(\kappa)}(r) \Phi_{j}^{(\nu)}(s)-\frac{1}{T_{0}} W_{1}^{(\kappa)}(r) T_{1}^{(\nu)}(s)\right]\right. \\
& \left.+H_{\alpha}^{(\kappa)}(r) v_{\alpha}^{(\nu)}(s)-\frac{1}{T_{0}} W_{2}^{(\kappa)}(r) T_{2}^{(\nu)}(s)\right\} d a \\
K_{\kappa \nu}(r, s) & =\int_{\Sigma}\left\{2 h \rho \ddot{w}_{j}^{(\kappa)}(r) w_{j}^{(\nu)}(s)+2 h J \ddot{\Phi}_{j}^{(\kappa)}(r) \Phi_{j}^{(\nu)}(s)\right. \\
& +\rho I \ddot{v}_{\alpha}^{(\kappa)}(r) v_{\alpha}^{(\nu)}(s)-\frac{k}{T_{0}}\left[2 h \widehat{T}_{1, \alpha}^{(\kappa)}(r) T_{1, \alpha}^{(\nu)}(s)\right. \\
& \left.\left.+I \widehat{T}_{2, \alpha}^{(\kappa)}(r) T_{2, \alpha}^{(\nu)}(s)+2 h \widehat{T}_{2}^{(\kappa)}(r) T_{2}^{(\nu)}(s)\right]\right\} d a
\end{aligned}
$$

for all $r, s \in \mathcal{T}$. Here, for convenience, we have suppressed the argument $\boldsymbol{x}$.

First we present a reciprocity relation which involves two processes at different instants.

Theorem 1. Let

$$
E_{\alpha \beta}(r, s)=\Pi_{\alpha \beta}(r, s)-K_{\alpha \beta}(r, s)
$$


for all $r, s \in \mathcal{T}$. Then

$$
E_{\alpha \beta}(r, s)=E_{\beta \alpha}(s, r) \quad(\alpha, \beta=1,2)
$$

Proof. We denote

$$
\begin{aligned}
W_{\kappa \nu}(r, s) & =2 h\left[\tau_{\beta j}^{(\kappa)}(r) \gamma_{\beta j}^{(\nu)}(s)+\tau_{3 \alpha}^{(\kappa)}(r) \gamma_{3 \alpha}^{(\nu)}(s)+\mu_{\beta j}^{(\kappa)}(r) \eta_{\beta j}^{(\nu)}(s)\right. \\
& \left.-\rho \zeta^{(\kappa)}(r) T_{1}^{(\nu)}(s)\right]+M_{\alpha \beta}^{(\kappa)}(r) \xi_{\alpha \beta}^{(\nu)}(s)-\rho \sigma^{(\kappa)}(r) T_{2}^{(\nu)}(s)
\end{aligned}
$$

In view of the constitutive equations (17) we find that

$$
W_{\kappa \nu}(r, s)=2 h W_{\kappa \nu}^{(1)}(r, s)+I W_{\kappa \nu}^{(2)}(r, s)
$$

where

$$
\begin{aligned}
W_{\kappa \nu}^{(1)}(r, s) & =\lambda \gamma_{\rho \rho}^{(\kappa)}(r) \gamma_{\eta \eta}^{(\nu)}(s)+(\mu+\kappa)\left[\gamma_{\alpha j}^{(\kappa)}(r) \gamma_{\alpha j}^{(\nu)}(s)+\gamma_{3 \alpha}^{(\kappa)}(r) \gamma_{3 \alpha}^{(\nu)}(s)\right] \\
& +\mu\left[\gamma_{\beta j}^{(\kappa)}(r) \gamma_{j \beta}^{(\nu)}(s)+\gamma_{3 \alpha}^{(\kappa)}(r) \gamma_{\alpha 3}^{(\nu)}(s)\right]+C_{1}\left[\eta_{\rho \rho}^{(\kappa)}(r) \gamma_{\beta \beta}^{(\nu)}(s)+\gamma_{\rho \rho}^{(\kappa)}(r) \eta_{\beta \beta}^{(\nu)}(s)\right] \\
& +C_{2}\left[\eta_{\alpha j}^{(\kappa)}(r) \gamma_{j \alpha}^{(\nu)}(s)+\gamma_{j \beta}^{(\kappa)}(r) \eta_{\beta j}^{(\nu)}(s)\right] \\
& +C_{3}\left[\eta_{\alpha j}^{(\kappa)}(r) \gamma_{\alpha j}^{(\kappa)}(s)+\gamma_{\alpha j}^{(\kappa)}(r) \eta_{\alpha j}^{(\nu)}(s)\right] \\
& +\alpha \eta_{\rho \rho}^{(\kappa)}(r) \eta_{\beta \beta}^{(\nu)}(s)+\beta \eta_{\alpha}^{(\kappa)}(r) \eta_{\alpha}^{(\nu)}(s) \\
& +\gamma \eta_{\alpha j}^{(\kappa)}(r) \eta_{\alpha j}^{(\nu)}(s)-b_{1}\left[T_{1}^{(\kappa)}(r) \gamma_{\rho \rho}^{(\nu)}(s)+T_{1}^{(\nu)}(s) \gamma_{\rho \rho}^{(\kappa)}(r)\right] \\
& -b_{2}\left[T_{1}^{(\kappa)}(r) \eta_{\rho \rho}^{(\nu)}(s)+T_{1}^{(\nu)}(s) \eta_{\rho \rho}^{(\kappa)}(r)\right]-a T_{1}^{(\kappa)}(r) T_{1}^{(\nu)}(s)
\end{aligned}
$$


and

$$
\begin{aligned}
W_{\kappa \nu}^{(2)}(r, s) & =\lambda \xi_{\rho \rho}^{(\kappa)}(r) \xi_{\alpha \alpha}^{(\nu)}(s)+(\mu+\kappa) \xi_{\alpha \beta}^{(\kappa)}(r) \xi_{\alpha \beta}^{(\nu)}(s) \\
& +\mu \xi_{\beta \alpha}^{(\kappa)}(r) \xi_{\alpha \beta}^{(\nu)}(s)-b_{1}\left[T_{2}^{(\kappa)}(r) \xi_{\rho \rho}^{(\nu)}(s)\right. \\
& \left.+T_{2}^{(\nu)}(s) \xi_{\rho \rho}^{(\kappa)}(r)\right]-a T_{2}^{(\kappa)}(r) T_{2}^{(\nu)}(s)
\end{aligned}
$$

From (32)-(34) we get

$$
W_{\kappa \nu}(r, s)=W_{\nu \kappa}(s, r)
$$

On the other hand, if we use the equations $(7),(9),(26),(17)$ and (26) we find that

$$
\begin{aligned}
W_{\kappa \nu}(r, s) & =2 h\left[F_{k}^{(\kappa)}(r) w_{k}^{(\nu)}(s)+G_{k}^{(\kappa)}(r) \Phi_{k}^{(\nu)}(s)-\frac{1}{T_{0}} W_{1}^{(\kappa)}(r) T_{1}^{(\nu)}(s)\right] \\
& +H_{\alpha}^{(\kappa)}(r) v_{\alpha}^{(\nu)}(s)-\frac{1}{T_{0}} W_{2}^{(\kappa)}(r) T_{2}^{(\nu)}(s)-2 h\left[\rho \ddot{w}_{k}^{(\kappa)}(r) w_{k}^{(\nu)}(s)\right. \\
& \left.+J \ddot{\Phi}_{k}^{(\kappa)}(r) \Phi_{k}^{(\nu)}(s)\right]-\rho I \ddot{v}_{\alpha}^{(\kappa)}(r) v_{\alpha}^{(\nu)}(s) \\
& +\left\{2 h\left[\tau_{\beta k}^{(\kappa)}(r) w_{k}^{(\nu)}(s)+\mu_{\beta k}^{(\kappa)}(r) \Phi_{k}^{(\nu)}(s)-\frac{1}{T_{0}} \widehat{\chi}_{\beta}^{(\kappa)}(r) T_{1}^{(\nu)}(s)\right]\right. \\
& \left.+M_{\beta \alpha}^{(\kappa)}(r) v_{\alpha}^{(\nu)}(s)-\frac{1}{T_{0}} \widehat{Q}_{\beta}^{(\kappa)}(r) T_{2}^{(\nu)}(s)\right\}_{, \beta} \\
& +\frac{k}{T_{0}}\left[2 h \widehat{T}_{1, \alpha}^{(\kappa)}(r) T_{1, \alpha}^{(\nu)}(s)+I \widehat{T}_{2, \alpha}^{(\kappa)}(r) T_{2, \alpha}^{(\nu)}(s)+2 h \widehat{T}_{2}^{(\kappa)}(r) T_{2}^{(\nu)}(s)\right]
\end{aligned}
$$

If we integrate (36) over $\Sigma$ and use (27)-(29) and the divergence theorem, then we obtain

$$
\int_{\Sigma} W_{\kappa \nu}(r, s) d a=E_{\kappa \nu}(r, s)
$$

From (35) and (37) we obtain the desired result. We introduce the notations

$$
\tau_{k}=\tau_{\alpha k} n_{\alpha} \mu_{k}=\mu_{\alpha k} n_{\alpha} M_{\alpha}=M_{\beta \alpha} n_{\beta} \quad \chi=\chi_{\alpha} n_{\alpha} Q=q_{\alpha} n_{\alpha}
$$


Theorem 2. Let $A=\left\{w_{k}, \Phi_{k}, v_{\beta}, T_{\beta}, \gamma_{i j}, \eta_{\beta k}, \xi_{\beta \alpha}, \zeta, \sigma, \tau_{i j}, \mu_{\alpha j}, M_{\alpha \beta}, \chi_{j}, Q_{\alpha}\right\}$ be a solution corresponding to the system of loading $\left\{F_{i}, G_{i}, H_{\alpha}, S_{\alpha}, w_{i}^{0}, \Phi_{i}^{0}, T_{\alpha}^{0}, v_{\alpha}^{0}, \vartheta_{j}^{0}, \omega_{j}^{0}, \eta_{\alpha}^{0}, \widetilde{\tau}_{j}\right.$, $\left.\widetilde{\mu}_{j}, \widetilde{M}_{\alpha}, \widetilde{\chi}, \widetilde{Q}\right\}$ and let

$$
\begin{aligned}
\Pi(r, s) & =\int_{\Sigma}\left[2 h F_{j}(r) w_{j}(s)+2 h G_{j}(r) \Phi_{j}(s)-2 h T_{0}^{-1} W_{1}(r) T_{1}(s)\right. \\
& \left.+H_{\alpha}(r) v_{\alpha}(s)-T_{0}^{-1} W_{2}(r) T_{2}(s)\right] d a \\
& +\int_{L}\left[2 h \tau_{j}(r) w_{j}(s)+2 h \mu_{j}(r) \Phi_{j}(s)-2 h T_{0}^{-1} \widehat{\chi}(r) T_{1}(s)\right. \\
& \left.+M_{\beta}(r) v_{\beta}(s)-T_{0}^{-1} \widehat{Q}(r) T_{2}(s)\right] d l
\end{aligned}
$$

for all $r, s \in \mathcal{T}$. Then

$$
\begin{aligned}
& \frac{d}{d t}\left\{\int_{\Sigma}\left(2 h \rho w_{j} w_{j}+2 h J \Phi_{j} \Phi_{j}+\rho I v_{\alpha} v_{\alpha}\right) d a\right. \\
& \left.+k T_{0}^{-1} \int_{0}^{t} \int_{\Sigma}\left(2 h \widehat{T}_{1, \alpha} \widehat{T}_{1, \alpha}+I \widehat{T}_{2, \alpha} \widehat{T}_{2, \alpha}+2 h \widehat{T}_{2}^{2}\right) d t d a\right\} \\
& =\int_{0}^{t}[\Pi(t-s, t+s)-\Pi(t+s, t-s)] d s \\
& +\int_{\Sigma}\left\{2 h \rho\left[\dot{w}_{j}(0) w_{j}(2 t)+\dot{w}_{j}(2 t) w_{j}(0)\right]+2 h J\left[\dot{\Phi}_{j}(0) \Phi_{j}(2 t)+\dot{\Phi}_{j}(2 t) \Phi_{j}(0)\right]\right. \\
& \left.+\rho I\left[\dot{v}_{\alpha}(0) v_{\alpha}(2 t)+\dot{v}_{\alpha}(2 t) v_{\alpha}(0)\right]\right\} d a
\end{aligned}
$$

Proof. In view of (30) we obtain

$$
\int_{0}^{t} E_{11}(t+s, t-s) d s=\int_{0}^{t} E_{11}(t-s, t+s) d s
$$


Let us apply this relation to the solution $A^{(1)}=A$. From (28), (29) and (39) we obtain

$$
\begin{aligned}
& \int_{0}^{t} E_{11}(t+s, t-s) d s=\int_{0}^{t} \Pi(t+s, t-s) d s-\int_{0}^{t} \int_{\Sigma}\left\{2 h \rho \ddot{w}_{j}(t+s) w_{j}(t-s)\right. \\
& +2 h J \ddot{\Phi}_{j}(t+s) \Phi_{j}(t-s)+\rho I \ddot{v}_{\alpha}(t+s) v_{\alpha}(t-s) \\
& -k T_{0}^{-1}\left[2 h \widehat{T}_{1, \alpha}(t+s) T_{1, \alpha}(t-s)\right. \\
& \left.\left.+I \widehat{T}_{2, \alpha}(t+s) T_{2, \alpha}(t-s)+2 h \widehat{T}_{2}(t+s) T_{2}(t-s)\right]\right\} d s d a
\end{aligned}
$$

and

$$
\begin{aligned}
& \int_{0}^{t} E_{11}(t-s, t+s) d s=\int_{0}^{t} \Pi(t-s, t+s) d s-\int_{0}^{t} \int_{\Sigma}\left\{2 h \rho \ddot{w}_{j}(t-s) w_{j}(t+s)\right. \\
& +2 h J \ddot{\Phi}_{j}(t-s) \Phi_{j}(t+s)+\rho I \ddot{v}_{\alpha}(t-s) v_{\alpha}(t+s) \\
& -k T_{0}^{-1}\left[2 h \widehat{T}_{1, \alpha}(t-s) T_{1, \alpha}(t+s)+I \widehat{T}_{2, \alpha}(t-s) T_{2, \alpha}(t+s)\right. \\
& \left.\left.+2 h \widehat{T}_{2}(t-s) T_{2}(t+s)\right]\right\} d s d a
\end{aligned}
$$

Clearly, if $p$ and $q$ are functions of class $C^{2}$ on $[0, \infty)$, then we have

$$
\begin{aligned}
& \int_{0}^{t} p(t+s) \dot{q}(t-s) d s=-q(0) p(2 t)+p(t) q(t)+\int_{0}^{t} \dot{p}(t+s) q(t-s) d s \\
& \int_{0}^{t} \ddot{p}(t+s) q(t-s) d s=\dot{p}(2 t) q(0)-\dot{p}(t) q(t)+\int_{0}^{t} \dot{q}(t-s) \dot{p}(t+s) d s \\
& \int_{0}^{t} \ddot{q}(t-s) p(t+s) d s=\dot{q}(t) p(t)-\dot{q}(0) p(2 t)+\int_{0}^{t} \dot{q}(t-s) \dot{p}(t+s) d s
\end{aligned}
$$

If we use these relations, then we can present (42) and (43) in a different form. Thus, 
the relation (42) can be expressed as

$$
\begin{aligned}
& \int_{0}^{t} E_{11}(t+s, t-s) d s=\int_{0}^{t} \Pi(t+s, t-s) d s-\int_{\Sigma}\left\{2 h \rho \left[\dot{w}_{j}(2 t) w_{j}(0)\right.\right. \\
& \left.-\dot{w}_{j}(t) w_{j}(t)+\int_{0}^{t} \dot{w}_{j}(t+s) \dot{w}_{j}(t-s) d s\right]+2 h J\left[\dot{\Phi}_{j}(2 t) \Phi_{j}(0)\right. \\
& \left.-\dot{\Phi}_{j}(t) \Phi_{j}(t)+\int_{0}^{t} \dot{\Phi}_{j}(t+s) \dot{\Phi}_{j}(t-s) d s\right] \\
& +\rho I\left[\dot{v}_{\alpha}(2 t) v_{\alpha}(0)-\dot{v}_{\alpha}(t) v_{\alpha}(t)+\int_{0}^{t} \dot{v}_{\alpha}(t+s) \dot{v}_{\alpha}(t-s) d s\right] \\
& -2 h k T_{0}^{-1}\left[\widehat{T}_{1, \alpha} \widehat{T}_{1, \alpha}+\int_{0}^{t} T_{1, \alpha}(t+s) \widehat{T}_{1, \alpha}(t-s) d s\right] \\
& -I k T_{0}^{-1}\left[\widehat{T}_{2, \alpha} \widehat{T}_{2, \alpha}+\int_{0}^{t} T_{2, \alpha}(t+s) \widehat{T}_{2, \alpha}(t-s) d s\right] \\
& \left.-2 h k T_{0}^{-1}\left[\widehat{T}_{2}^{2}+\int_{0}^{t} T_{2}(t+s) \widehat{T}_{2}(t-s) d s\right]\right\} d a
\end{aligned}
$$

The relation (43) can be transformed in a similar way. By using (41), (43) and (44) we obtain (40)

Theorem 2 forms the basis of the following uniqueness result.

Theorem 3. Assume that $\rho, J$ and $k$ are strictly positive and a is different from zero. Then the boundary-initial-value problem has at most one solution.

Proof. If there are two solutions, then their difference $A$ coresponds to null data. In view of (40) and the initial conditions we get

$$
\begin{aligned}
& \int_{\Sigma}\left(2 h \rho w_{j} w_{j}+2 h J \Phi_{j} \Phi_{j}+\rho I v_{\alpha} v_{\alpha}\right) d a \\
& +\frac{k}{T_{0}} \int_{0}^{t} \int_{\Sigma}\left(2 h \widehat{T}_{1, \alpha} \widehat{T}_{1, \alpha}+I \widehat{T}_{2, \alpha} \widehat{T}_{2, \alpha}+2 h \widehat{T}_{2}^{2}\right) d t d a=0
\end{aligned}
$$


By the hypotheses of theorem and (45) we find that

$$
w_{i}=0 \Phi_{i}=0 v_{\alpha}=0 \widehat{T}_{1, \alpha}=0 \widehat{T}_{2}=0 \text { on } \Sigma \times[0, \infty)
$$

From (46) we obtain $T_{1, \alpha}=0$ on $\Sigma \times \mathcal{T}$ so that $\chi_{\alpha}=0$ on $\Sigma \times \mathcal{T}$ and $T_{1}(x, t)=y(t)$, $x \in \Sigma, t \in[0, \infty)$. The constitutive equations (17) and (46) imply that $\rho \zeta=a y$. The energy equation (11) implies that $a T_{0} \dot{y}=0$. Since $a$ and $T_{0}$ are different from zero we conclude that $y=$ constant on $\Sigma \times \mathcal{T}$. The initial conditions imply that $y(0)=0$ so that $T_{1}=0$ on $\Sigma \times I$. From (46) we get also that $T_{2}=0$. The proof is complete.

The method to obtain the uniqueness result has been given by Brun [18].

With the help of Theorem 1 we obtain the following reciprocity theorem.

Theorem 4. Let $A^{(\alpha)}$ be a solution corresponding to the external data system $\mathcal{L}^{(\alpha)}$, $(\alpha=1,2)$. Then

$$
\begin{aligned}
& \int_{\Sigma}\left[2 h \mathcal{F}_{i}^{(1)} * w_{i}^{(2)}+2 h \mathcal{G}_{i}^{(1)} * \Phi_{i}^{(2)}+\mathcal{H}_{\alpha}^{(1)} * v_{\alpha}^{(2)}\right. \\
& \left.-2 h T_{0}^{-1} g * W_{1}^{(1)} * T_{1}^{(2)}-T_{0}^{-1} g * W_{2}^{(1)} * T_{2}^{(2)}\right] d a \\
& +\int_{\Gamma} g *\left[2 h \tau_{j}^{(1)} * w_{j}^{(2)}+2 h \mu_{j}^{(1)} * \Phi_{j}^{(2)}-2 h T_{0}^{-1} f * \chi^{(1)} * T_{1}^{(2)}+M_{\beta}^{(1)} * v_{\beta}^{(2)}\right. \\
& \left.-T_{0}^{-1} f * Q^{(1)} * T_{2}^{(2)}\right] d l=\int_{\Sigma}\left[2 h \mathcal{F}^{(2)} * w_{i}^{(1)}+2 h \mathcal{G}_{i}^{(2)} * \Phi_{i}^{(1)}\right. \\
& \left.+\mathcal{H}_{\alpha}^{(2)} * v_{\alpha}^{(1)}-2 h T_{0}^{-1} g * W_{1}^{(2)} * T_{1}^{(1)}-T_{0}^{-1} g * W_{2}^{(2)} * T_{2}^{(1)}\right] d a \\
& +\int_{\Gamma} g *\left[2 h \tau_{j}^{(2)} * w_{j}^{(1)}+2 h \mu_{j}^{(2)} * \Phi_{j}^{(1)}-2 h T_{0}^{-1} f * \chi^{(2)} * T_{1}^{(1)}\right. \\
& \left.+M_{\beta}^{(2)} * v_{\beta}^{(1)}-T_{0}^{-1} f * Q^{(2)} * T_{2}^{(1)}\right] d l
\end{aligned}
$$


where

$$
\begin{aligned}
& \mathcal{F}_{i}^{(\alpha)}=g * F_{i}^{(\alpha)}+\rho\left(t \vartheta_{i}^{0(\alpha)}+w_{i}^{0(\alpha)}\right) \\
& \mathcal{G}_{i}^{(\alpha)}=g * G_{i}^{(\alpha)}+J\left(t \omega_{i}^{0(\alpha)}+\Phi_{i}^{0(\alpha)}\right) \mathcal{H}_{\rho}^{(\alpha)}=g * H_{\rho}^{(\alpha)}+\rho I\left(t \eta_{\rho}^{0(\alpha)}+v_{\rho}^{0(\alpha)}\right)
\end{aligned}
$$

Proof. Let us take in $(30), r=\tau$ and $s=t-\tau$. If we integrate with respect to $\tau$ from 0 to $t$, then with the aid of (28) and (29) we obtain

$$
\begin{aligned}
& \int_{\Sigma}\left[2 h F_{j}^{(1)} * w_{j}^{(2)}+2 h G_{j}^{(1)} * \Phi_{j}^{(2)}-2 h T_{0}^{-1} W_{1}^{(1)} * T_{1}^{(2)}\right. \\
& \left.+H_{\alpha}^{(1)} * v_{\alpha}^{(2)}-T_{0}^{-1} W_{2}^{(1)} * T_{2}^{(2)}\right] d a \\
& \int_{\Gamma}\left[2 h \tau_{j}^{(1)} * w_{j}^{(2)}+2 h \mu_{j}^{(1)} * \Phi_{j}^{(2)}-2 h T_{0}^{-1} \widehat{\chi}^{(1)} * T_{1}^{(2)}\right. \\
& \left.+M_{\beta}^{(1)} * v_{\beta}^{(2)}-T_{0}^{-1} \widehat{Q}^{(1)} * T_{2}^{(2)}\right] d l \\
& -\int_{\Sigma}\left\{2 h \rho \ddot{w}_{j}^{(1)} * w_{j}^{(2)}+2 h J \ddot{\Phi}_{j}^{(1)} * \Phi_{j}^{(2)}+\rho I \ddot{v}_{\alpha}^{(1)} * v_{\alpha}^{(2)}\right. \\
& \left.-k T_{0}^{-1}\left[2 h \widehat{T}_{1, \alpha}^{(1)} * T_{1, \alpha}^{(2)}+I \widehat{T}_{2, \alpha}^{(1)} * T_{2, \alpha}^{(2)}+2 h \widehat{T}_{2}^{(1)} * T_{2}^{(2)}\right]\right\} d a \\
& =\int_{\Sigma}\left[2 h F_{j}^{(2)} * w_{j}^{(1)}+2 h G_{j}^{(2)} * \Phi_{j}^{(1)}-2 h T_{0}^{-1} W_{1}^{(2)} * T_{1}^{(1)}\right. \\
& \left.+H_{\alpha}^{(2)} * v_{\alpha}^{(1)}-T_{0}^{-1} W_{2}^{(2)} * T_{2}^{(1)}\right] d a \\
& +\int_{\Gamma}\left[2 h \tau_{j}^{(2)} * w_{j}^{(1)}+2 h \mu_{j}^{(2)} * \Phi_{j}^{(1)}-2 h T_{0}^{-1} \widehat{\chi}^{(2)} * T_{1}^{(1)}\right. \\
& \left.+M_{\beta}^{(2)} * v_{\beta}^{(1)}-T_{0}^{-1} \widehat{Q}^{(2)} * T_{2}^{(1)}\right] d l \\
& -\int_{\Sigma}\left\{2 h \rho \ddot{w}_{j}^{(2)} * w_{j}^{(1)}+2 h J \ddot{\Phi}_{j}^{(2)} * \Phi_{j}^{(1)}+\rho I \ddot{v}_{\alpha}^{(2)} * v_{\alpha}^{(1)}\right. \\
& \left.-k T_{0}^{-1}\left[2 h \widehat{T}_{1, \alpha}^{(2)} * T_{1, \alpha}^{(1)}+I \widehat{T}_{2, \alpha}^{(2)} * T_{2, \alpha}^{(1)}+2 h \widehat{T}_{2}^{(2)} * T_{2}^{(1)}\right]\right\}
\end{aligned}
$$


We note that

$$
g * \ddot{w}_{j}^{(\alpha)}=w_{j}^{(\alpha)}-t \vartheta_{j}^{0(\alpha)}-w_{j}^{0(\alpha)}
$$

If we take the convolution of the relation (49) with $g$ and use (48) and (50), then we obtain (47)

The method to obtain this reciprocal theorem has been established in $[19,20]$.

\section{INSTABILITY OF SOLUTIONS}

In this section we suppose that the body forces and heat supply are absent and we consider the following boundary conditions

$$
w_{j}=0 \quad \Phi_{j}=0 v_{\alpha}=0 T_{\alpha}=0 \text { on } \Gamma \times \mathcal{T}
$$

We assume that the coefficients $\rho, I, c$ and $k$ are strictly positive.

The aim of this section is to study the instability of solutions to the equations (18) with the initial conditions (19) and the boundary conditions (51). The method used is strongly based on the choice of a special function [21]. Before to introduce this functions we shall establish some preliminaries. If we integrate with respect to the time the last two equations from (18), then we get

$$
\begin{aligned}
& \frac{k}{T_{0}} \int_{0}^{t} \Delta T_{1} d s-b_{1} w_{\alpha, \alpha}-b_{2} \Phi_{\alpha, \alpha}-a T_{1}=-\rho \zeta^{0} \\
& \frac{k I}{T_{0}} \int_{0}^{t} \Delta T_{2} d s-\frac{2 h k}{T_{0}} \int_{0}^{t} T_{2} d s-b_{1} I v_{\alpha, \alpha}-a I T_{2}=-\rho \sigma^{0}
\end{aligned}
$$

where $\rho \zeta^{0}$ and $\rho \sigma^{0}$ have been defined in (25). We denote by $P_{\alpha}\left(x_{1}, x_{2}\right)$ the functions 
which satisfy the equations

$$
k \Delta P_{1}=\rho \zeta^{0} T_{0} \quad k\left(I \Delta P_{2}-2 h P_{2}\right)=\rho \sigma^{0} T_{0} \text { on } \Sigma
$$

and the conditions

$$
P_{\alpha}=0 \text { on } \Gamma
$$

If we define the functions $z_{\alpha}$ by

$$
z_{\alpha}=P_{\alpha}+\int_{0}^{t} T_{\alpha} d s
$$

then we find that $z_{\alpha}$ satisfy the equations

$$
\begin{aligned}
& \frac{k}{T_{0}} \Delta z_{1}-b_{1} w_{\alpha, \alpha}-b_{2} \Phi_{\alpha, \alpha}-a T_{1}=0 \\
& \frac{k}{T_{0}}\left(I \Delta z_{2}-2 h z_{2}\right)-I\left(b_{1} v_{\alpha, \alpha}+a T_{2}\right)=0
\end{aligned}
$$

By performing calculations similar to those used to prove Theorem 2, we can obtain the following energy identity

$$
\begin{aligned}
E(t) & =\int_{\Sigma}\left(2 h \rho \dot{w}_{i} \dot{w}_{i}+2 h J \dot{\Phi}_{i} \dot{\Phi}_{i}+\rho I \dot{v}_{\alpha} \dot{v}_{\alpha}+2 h a T_{1}^{2}+I a T_{2}^{2}\right) d a \\
& +2 h \int_{\Sigma}\left(\lambda \gamma_{\rho \rho} \gamma_{\eta \eta}+(\mu+\kappa)\left(\gamma_{\alpha j} \gamma_{\alpha j}+\gamma_{3 \alpha} \gamma_{3 \alpha}\right)\right. \\
& +\mu\left(\gamma_{\beta j} \gamma_{j \beta}+\gamma_{3 \alpha} \gamma_{\alpha 3}\right)+2 C_{1} \eta_{\alpha \alpha} \gamma_{\beta \beta}+2 C_{2} \eta_{\alpha j} \gamma_{j \alpha} \\
& \left.+2 C_{3} \eta_{\alpha j} \gamma_{\alpha j}+\alpha \eta_{\alpha \alpha} \eta_{\beta \beta}+\beta \eta_{\alpha j} \eta_{j \alpha}+\gamma \eta_{\alpha j} \eta_{\alpha j}\right) d a \\
& +I \int_{\Sigma}\left(\lambda \xi_{\alpha \alpha} \xi_{\beta \beta}+(\mu+\kappa) \xi_{\alpha \beta} \xi_{\alpha \beta}+\mu \xi_{\alpha \beta} \xi_{\beta \alpha}\right) d a \\
& +\frac{2 k}{T_{0}} \int_{0}^{t} \int_{\Sigma}\left(2 h T_{1, \alpha} T_{1, \alpha}+I T_{2, \alpha} T_{2, \alpha}+2 h T_{2}^{2}\right) d a d s=E(0)
\end{aligned}
$$


We now define the function $F_{h, \omega}$ on $\mathcal{T}$ by

$$
\begin{aligned}
F_{h, \omega}(t) & =\int_{\Sigma}\left(2 h \rho w_{i} w_{i}+2 h J \Phi_{i} \Phi_{i}+\rho I v_{\alpha} v_{\alpha}\right) d a \\
& +\frac{k}{T_{0}} \int_{0}^{t} \int_{\Sigma}\left(2 h z_{1, \alpha} z_{1, \alpha}+I z_{2, \alpha} z_{2, \alpha}+2 h z_{2}^{2}\right) d a d s+h(t+w)^{2}
\end{aligned}
$$

where $h$ and $w$ are two positive constants that will be determined later. From (58) we find that

$$
\begin{aligned}
\dot{F}_{h, w}(t) & =2 \int_{\Sigma}\left(2 h \rho w_{i} \dot{w}_{i}+2 h J \Phi_{i} \dot{\Phi}_{i}+\rho I v_{\alpha} \dot{v}_{\alpha}\right) d a \\
& +\frac{2 k}{T_{0}} \int_{0}^{t} \int_{\Sigma}\left(2 h z_{1, \alpha} \dot{z}_{1, \alpha}+I z_{2, \alpha} \dot{z}_{2, \alpha}+2 h z_{2} \dot{z}_{2}\right) d a d s \\
& +2 h(t+w)-\frac{k}{T_{0}} \int_{\Sigma}\left(2 h P_{1, \alpha} P_{1, \alpha}+I P_{2, \alpha} P_{2, \alpha}+2 h P_{2}^{2}\right) d a \\
\ddot{F}_{h, w}(t) & =2 \int_{\Sigma}\left(2 h \rho\left(w_{i} \ddot{w}_{i}+\dot{w}_{i} \dot{w}_{i}\right)+2 h J\left(\Phi_{i} \ddot{\Phi}_{i}+\dot{\Phi}_{i} \dot{\Phi}_{i}\right)\right. \\
& \left.+\rho I\left(v_{\alpha} \ddot{v}_{\alpha}+\dot{v}_{\alpha} \dot{v}_{\alpha}\right)\right) d a \\
& +\frac{2 k}{T_{0}} \int_{\Sigma}\left(2 h z_{1, \alpha} \dot{z}_{1, \alpha}+I z_{2, \alpha} \dot{z}_{2, \alpha}+2 h z_{2} \dot{z}_{2}\right) d a+2 h
\end{aligned}
$$


By using the basic equations and the divergence theorem, we get

$$
\begin{aligned}
\ddot{F}_{h, \omega}(t) & =2 \int_{\Sigma}\left(2 h \rho \dot{w}_{i} \dot{w}_{i}+2 h J \dot{\Phi}_{i} \dot{\Phi}_{i}+\rho I \dot{v}_{\alpha} \dot{v}_{\alpha}\right) d a \\
& -2 \int_{\Sigma} 2 h\left\{\lambda \gamma_{\rho \rho} \gamma_{\eta \eta}+(\mu+\kappa)\left[\gamma_{\alpha j} \gamma_{\alpha j}+\gamma_{3 \alpha} \gamma_{3 \alpha}\right]+\mu\left[\gamma_{\beta j} \gamma_{j \beta}+\gamma_{3 \alpha} \gamma_{\alpha 3}\right]\right. \\
& +2 C_{1} \eta_{\rho \rho} \gamma_{\beta \beta}+2 C_{2} \eta_{\alpha j} \gamma_{j \alpha}+2 C_{3} \eta_{\alpha j} \gamma_{\alpha j}+\alpha \eta_{\alpha \alpha} \eta_{\beta \beta} \\
& \left.+\beta \eta_{\alpha j} \eta_{j \alpha}+\gamma \eta_{\alpha j} \eta_{\alpha j}\right\} d a \\
& -2 \int_{\Sigma} I\left\{\lambda \xi_{\alpha \alpha} \xi_{\beta \beta}+(\mu+\kappa) \xi_{\alpha \beta} \xi_{\alpha \beta}+\mu \xi_{\alpha \beta} \xi_{\beta \alpha}\right\} d a \\
& -2 \int_{\Sigma} 2 h\left(\frac{k}{T_{0}} \Delta z_{1}-b_{1} \gamma_{\rho \rho}-b_{2} \eta_{\rho \rho}\right) \dot{z}_{1} d a \\
& -2 \int_{\Sigma}\left\{\frac{k}{T_{0}}\left(I \Delta z_{2}-2 h z_{2}\right)-b_{1} I \xi_{\alpha \alpha}\right\} \dot{z}_{2} d a+2 h
\end{aligned}
$$

In view of the field equations, we find that

$$
\begin{aligned}
\ddot{F}_{h, \omega}(t) & =2 \int_{\Sigma}\left(2 h \rho \dot{w}_{i} \dot{w}_{i}+2 h J \dot{\Phi}_{i} \dot{\Phi}_{i}+\rho I \dot{v}_{\alpha} \dot{v}_{\alpha}\right) d l \\
& -2 \int_{\Sigma} 2 h\left\{\lambda \gamma_{\rho \rho} \gamma_{\eta \eta}+(\mu+\kappa)\left[\gamma_{\alpha j} \gamma_{\alpha j}+\gamma_{3 \alpha} \gamma_{3 \alpha}\right]+\mu\left[\gamma_{\beta j} \gamma_{\beta j}+\gamma_{3 \alpha} \gamma_{3 \alpha}\right]\right. \\
& +2 C_{1} \eta_{\rho \rho} \gamma_{\beta \beta}+2 C_{2} \eta_{\alpha j} \gamma_{j \alpha}+2 C_{3} \eta_{\alpha j} \gamma_{\alpha j}+\alpha \eta_{\alpha \alpha} \eta_{\beta \beta} \\
& \left.+\beta \eta_{\alpha j} \eta_{j \alpha}+\gamma \eta_{\alpha j} \eta_{\alpha j}\right\} d a \\
& -2 \int_{\Sigma} I\left\{\lambda \xi_{\alpha \alpha} \xi_{\beta \beta}+(\mu+\kappa) \xi_{\alpha \beta} \xi_{\alpha \beta}+\mu \xi_{\alpha \beta} \xi_{\beta \alpha}\right\} d a \\
& -2 \int_{\Sigma}\left(2 h a T_{1}^{2}+I a T_{2}^{2}\right) d a+2 h
\end{aligned}
$$

From (57) and (61) we obtain

$$
\begin{aligned}
\ddot{F}_{h, \omega}(t) & =4 \int_{\Sigma}\left(2 h \dot{w}_{i} \dot{w}_{i}+2 h J \dot{\Phi}_{i} \dot{\Phi}_{i}+\rho I \dot{v}_{\alpha} \dot{v}_{\alpha}\right) d a \\
& +\frac{4 k}{T_{0}} \int_{0}^{t} \int_{\Sigma}\left(2 h T_{1, \alpha} T_{1, \alpha}+I T_{2, \alpha} T_{2, \alpha}+2 h T_{2}^{2}\right) d a d s-2(E(0)-h)
\end{aligned}
$$


We introduce the notation

$$
\nu=\frac{2 k}{T_{0}} \int_{\Sigma}\left(2 h P_{1, \alpha} P_{1, \alpha}+I P_{2, \alpha} P_{2, \alpha}+2 h P_{2}^{2}\right) d a
$$

From (58), (59) and (62) we obtain the inequality

$$
F_{h, \omega} \ddot{F}_{h, \omega}-\left(\dot{F}_{h, \omega}-\frac{\nu}{2}\right)^{2} \geq 2(h+E(0)) F_{h, \omega}
$$

In the case $E(0)<0$, we select $h=-E(0)$ and we take $\omega$ so large to guarantee that $\dot{F}_{h, \omega}(0)>\nu$. Following [22] we obtain

$$
F_{h, \omega}(t) \geq \frac{F_{h, \omega}(0) \dot{F}_{h, \omega}(0)}{\dot{F}_{h, \omega}(0)-\nu} \exp \left(\frac{\dot{F}_{h, \omega}(0)-\nu}{F_{h, \omega}(0)} t\right)-\frac{\nu F_{h, \omega}(0)}{\dot{F}_{h, \omega}(0)-\nu}
$$

The inequality (65) gives the exponential growth of the solutions. We have obtained the following result

Theorem 5. Assume that the coefficients $\rho, J, c$ and $k$ are strictly positive. If $E(0)<0$, then the solution becomes unbounded in an exponential way.

\section{AN EXISTENCE RESULT}

Throughout this section we use a semigroup approach (see [22]) to derive an existence theorem in the dynamical theory with the boundary conditions (51). We introduce the 
notation

$$
\begin{aligned}
2 W & =2 h\left[\lambda \gamma_{\rho \rho} \gamma_{\nu \nu}+(\mu+\kappa)\left(\gamma_{\alpha j} \gamma_{\alpha j}+\gamma_{3 \alpha} \gamma_{\alpha 3}\right)\right. \\
& +\mu\left(\gamma_{\beta j} \gamma_{j \beta}+\gamma_{3 \alpha} \gamma_{\alpha 3}\right)+2 C_{1} \eta_{\alpha \alpha} \gamma_{\beta \beta}+2 C_{2} \eta_{\alpha j} \gamma_{j \alpha} \\
& \left.+2 C_{3} \eta_{\alpha j} \gamma_{\alpha j}+\alpha \eta_{\rho \rho} \eta_{\nu \nu}+\beta \eta_{\nu j} \eta_{j \nu}+\gamma \eta_{\rho j} \eta_{\rho j}\right] \\
& +I\left[\lambda \xi_{\alpha \alpha} \xi_{\beta \beta}+(\mu+\kappa) \xi_{\alpha \beta} \xi_{\alpha \beta}+\mu \xi_{\alpha \beta} \xi_{\beta \alpha}\right]
\end{aligned}
$$

In what follows we assume that

(i) $\rho, J, c$ and $k$ are strictly positive;

(ii) there exists a positive constant $C$ such that

$$
2 W \geq C\left(\gamma_{\alpha j} \gamma_{\alpha j}+\gamma_{3 \alpha} \gamma_{3 \alpha}+\eta_{\alpha j} \eta_{\alpha j}+\xi_{\alpha \beta} \xi_{\alpha \beta}\right)
$$

for every $\gamma_{\alpha j}, \eta_{\alpha j}$ and $\xi_{\alpha \beta}$.

Let $\dot{w}_{j}=z_{j}, \dot{\Phi}=\Psi_{j}, \dot{v}_{j}=y_{\alpha}$ and define $\mathcal{Z}=\left\{\omega=\left(w_{j}, z_{j}, \Phi_{j}, \Psi_{j}, v_{\alpha}, y_{\alpha}, T_{\alpha}\right)\right.$; $\left.w_{j}, \Phi_{j}, v_{\alpha} \in W_{0}^{1,2} ; z_{j}, \Psi_{j}, y_{\alpha}, T_{\alpha} \in L_{2}\right\}$, where $W_{0}^{1,2}$ and $L^{2}$ are the well-known Hilbert 
spaces. Let

$$
\begin{aligned}
A_{i} \omega & =z_{i}, B_{\alpha} \omega=\frac{1}{\rho}\left[(\mu+\kappa) \Delta w_{\alpha}+(\lambda+\mu) w_{\rho, \rho \alpha}+C_{3} \Delta \Phi_{\alpha}\right. \\
& \left.+\left(C_{1}+C_{2}\right) \Phi_{\rho, \rho \alpha}+\kappa \varepsilon_{3 \alpha \beta} \Phi_{3, \beta}-b_{1} T_{1, \alpha}\right] \\
B_{3} \omega & =\frac{1}{\rho}\left[(\mu+\kappa) \Delta w_{3}+C_{3} \Delta \Phi_{3}+\kappa \varepsilon_{3 \alpha \beta} \Phi_{\beta, \alpha}+\mu v_{\beta, \beta}\right] \\
K_{i} \omega & =\Psi_{i}, D_{\nu} \omega=\frac{1}{J}\left[C_{3} \Delta w_{\nu}+\left(C_{1}+C_{2}\right) w_{\rho, \rho \nu}\right. \\
& +\gamma \Delta \Phi_{\nu}+(\alpha+\beta) \Phi_{\rho, \rho \nu}+\kappa \varepsilon_{\nu \rho 3}\left(w_{3, \rho}-v_{\rho}\right) \\
& \left.+2\left(C_{3}-C_{2}\right) \varepsilon_{3 \alpha \beta} \Phi_{3, \beta}-2 \kappa \Phi_{\alpha}-b_{2} T_{1, \alpha}\right] \\
D_{3} \omega & =\frac{1}{J}\left[C_{3} \Delta w_{3}+\gamma \Delta \Phi_{3}+\kappa \varepsilon_{3 \rho \alpha} w_{\alpha, \rho}\right. \\
& \left.+2 \varepsilon_{3 \alpha \beta}\left(C_{3}-C_{2}\right) \Phi_{\beta, \alpha}+C_{2} v_{\alpha, \alpha}-2 \kappa \Phi_{3}\right] \\
E_{\alpha} \omega & =y_{\alpha}, \mathcal{F}_{\alpha} \omega=\frac{1}{\rho I}\left\{I \left[(\mu+\kappa) \Delta v_{\alpha}+(\lambda+\mu) v_{\rho, \rho \alpha}\right.\right. \\
& \left.-b_{1} T_{2, \alpha}\right]-2 h\left[\mu w_{3, \alpha}+C_{2} \Phi_{3, \alpha}+\kappa \varepsilon_{3 \beta \alpha} \Phi_{\beta}+(\mu+\kappa) v_{\alpha}\right\} \\
G_{1} \omega & =\frac{1}{c}\left(k \Delta T_{1}-T_{0} b_{1} z_{\alpha, \alpha}-T_{0} b_{2} \Psi_{\alpha, \alpha}\right) \\
G_{2} \omega & =\frac{1}{c}\left[I\left(k \Delta T_{2}-T_{0} b_{1} y_{\alpha, \alpha}\right)-2 h k T_{2}\right]
\end{aligned}
$$

We introduce the operator $\mathcal{A}$ on $\mathcal{Z}$ defined by

$$
\mathcal{A} \omega=\left(A_{i} \omega, B_{i} \omega, K_{i} \omega, D_{i} \omega, E_{\alpha} \omega, \mathcal{F}_{\alpha} \omega, G_{\alpha} \omega\right)
$$

with the domain

$$
\mathcal{D}=\left\{\omega=\left(w_{i}, z_{i}, \Phi_{i}, \Psi_{i}, v_{\alpha}, y_{\alpha}, T_{\alpha}\right) \in \mathcal{Z} ; \mathcal{A} \omega \in \mathcal{Z}\right\}
$$

We note that the domain of the operator is dense. The boundary-initial-value problem 
characterized by the relations (18), (19) and (51) can be reduced to the following abstract equation in the space $\mathcal{Z}$,

$$
\frac{d \omega}{d t}=\mathcal{A} \omega+\mathcal{B}, \omega(0)=\omega_{0}
$$

where the vectors $\mathcal{B}$ and $\omega_{0}$ are defined by

$$
\begin{aligned}
\mathcal{B} & =\left(0,0,0, F_{i} / \rho, 0,0,0, G_{i} / J, 0,0, H_{\alpha} /(\rho I), S_{\alpha} / c\right\} \\
\omega_{0} & =\left(w_{i}^{0}, \vartheta_{j}^{0}, \Phi_{j}^{0}, \omega_{j}^{0}, v_{\alpha}^{0}, \eta_{\alpha}^{0}, T_{\alpha}^{0}\right)
\end{aligned}
$$

Let $\omega=\left(w_{j}, z_{j}, \Phi_{j}, \Psi_{j}, v_{\alpha}, y_{\alpha}, T_{\alpha}\right)$ and $\omega^{\prime}=\left(w_{j}^{\prime}, z_{j}^{\prime}, \Phi_{j}^{\prime}, \Psi_{j}^{\prime}, v_{\alpha}^{\prime}, y_{\alpha}^{\prime}, T_{\alpha}^{\prime}\right)$. We introduce the inner product

$$
\begin{aligned}
<\omega, \omega^{\prime}> & =\int_{\Sigma}\left(2 h \rho z_{i} z_{i}^{\prime}+2 h J \Psi_{i} \Psi_{i}^{\prime}+\rho I v_{\alpha} v_{\alpha}^{\prime}\right. \\
& \left.+2 h a T_{1}^{2}+I a T_{2}^{2}\right) d a+\int_{\Sigma} 2 W^{*} d a
\end{aligned}
$$

where

$$
\begin{aligned}
W^{*} & =2 h\left[\lambda \gamma_{\rho \rho} \gamma_{\eta \eta}^{\prime}+(\mu+\kappa)\left(\gamma_{\alpha j} \gamma_{\alpha j}^{\prime}+\gamma_{3 \alpha} \gamma_{3 \alpha}^{\prime}\right)\right. \\
& +\mu\left(\gamma_{\beta j} \gamma_{\beta j}^{\prime}+\gamma_{3 \alpha} \gamma_{3 \alpha}^{\prime}\right)+C_{1}\left(\eta_{\alpha \alpha} \gamma_{\beta \beta}^{\prime}+\eta_{\alpha \alpha}^{\prime} \gamma_{\beta \beta}\right) \\
& +C_{2}\left(\eta_{\alpha j} \gamma_{j \alpha}^{\prime}+\eta_{\alpha j}^{\prime} \gamma_{j \alpha}\right)+C_{3}\left(\eta_{\alpha j} \gamma_{\alpha j}^{\prime}+\gamma_{\alpha j} \eta_{\alpha j}^{\prime}\right) \\
& \left.+\alpha \eta_{\alpha \alpha} \eta_{\beta \beta}^{\prime}+\beta \eta_{\alpha j} \eta_{j \alpha}^{\prime}+\gamma \eta_{\alpha j} \eta_{\alpha j}^{\prime}\right]+I\left[\lambda \xi_{\rho \rho} \xi_{\alpha \alpha}^{\prime}\right. \\
& \left.+(\mu+\kappa) \xi_{\alpha \beta} \xi_{\alpha \beta}^{\prime}+\mu \xi_{\alpha \beta} \xi_{\beta \alpha}^{\prime}\right] \\
\gamma_{\alpha j}^{\prime} & =w_{j, \alpha}^{\prime}+\varepsilon_{j \alpha k} \Phi_{k}^{\prime} \gamma_{3 \alpha}^{\prime}=v_{\alpha}^{\prime}+\varepsilon_{3 \beta \alpha} \Phi_{\beta}^{\prime} \eta_{\alpha k}^{\prime}=\Phi_{k, \alpha}^{\prime} \xi_{\alpha \beta}^{\prime}=v_{\beta, \alpha}^{\prime} .
\end{aligned}
$$

This product defines the norm

$$
\|\omega\|^{2}=\int_{\Sigma}\left(2 h \rho z_{i} z_{i}+2 h J \Psi_{i} \Psi_{i}+\rho I v_{\alpha} v_{\alpha}+2 h a T_{1}^{2}+I a T_{2}^{2}\right) d a+2 \int_{\Sigma} W d a
$$


where $W$ is given by (67). The norm (75) is equivalent to the usual norm in $\mathcal{Z}$.

Lemma 2. Assume that the hypotheses (i) and (ii) hold. Then, for every $\omega \in \mathcal{D}$, we have

$$
<\mathcal{A} \omega, \omega>\leq 0
$$

Proof. If we use the relations (68), (69), the divergence theorem and the conditions (51) we get

$$
<\mathcal{A} \omega, \omega>=-\frac{k}{T_{0}} \int_{\Sigma}\left(2 h T_{1, \alpha} T_{1, \alpha}+I T_{2, \alpha} T_{2, \alpha}+2 h T_{2}^{2}\right) d a
$$

In view of (i) we obtain the desired result.

Lemma 3. Let $\rho(\mathcal{A})$ be the resolvent of the operator $\mathcal{A}$. Then $0 \in \rho(\mathcal{A})$.

Proof. We have to prove that the equation

$$
\mathcal{A} \omega=\mathcal{F}
$$

has a solution $\omega=\left(w_{i}, z_{i}, \Phi_{i}, \Psi_{i}, v_{\alpha}, y_{\alpha}, T_{\alpha}\right) \in \mathcal{D}$ for any $\mathcal{F}=\left(f_{1}, f_{2}, \ldots, f_{18}\right) \in \mathcal{Z}$. The equation (77) can be written in the form

$$
\begin{aligned}
& z_{i}=f_{i} B_{i} \omega=f_{3+i} \Psi_{j}=f_{6+j} D_{i} \omega=f_{9+i} \\
& y_{\alpha}=f_{12+\alpha} \mathcal{F}_{\alpha} \omega=f_{14+\alpha} G_{\alpha} \omega=f_{16+\alpha}
\end{aligned}
$$

From (78) we find that $z_{j}, \Psi_{j}, y_{\alpha} \in W_{0}^{1,2}$. The last two equations from (78) can be 
written as

$$
\begin{aligned}
& k \Delta T_{1}=c f_{17}+T_{0} b_{1} f_{\alpha, \alpha}+T_{0} b_{2} f_{6+\alpha, \alpha} \\
& k \Delta T_{2}-\frac{2 h k}{I} T_{2}=\frac{c}{I} f_{18}+T_{0} b_{1} f_{12+\alpha, \alpha}
\end{aligned}
$$

We note that we can find the functions $T_{1}, T_{2} \in W_{0}^{1,2}$ which satisfy (79). The system (78) reduces to

$$
\mathcal{L}_{i} u=g_{i} \mathcal{M}_{i} u=g_{3+i} \mathcal{H}_{\alpha} u=g_{6+\alpha}
$$

where $u=\left(w_{i}, \Phi_{i}, v_{\alpha}\right)$, and $\mathcal{L}_{j}, \mathcal{M}_{j}, \mathcal{H}_{\alpha}$ and $g_{s}$ are defined by

$$
\begin{aligned}
\mathcal{L}_{\alpha} u & =\frac{1}{\rho}\left[(\mu+\kappa) \Delta w_{\alpha}+(\lambda+\mu) w_{\rho, \rho \alpha}+C_{3} \Delta \Phi_{\alpha}\right. \\
& \left.+\left(C_{1}+C_{2}\right) \Phi_{\rho, \rho \alpha}+\kappa \varepsilon_{3 \alpha \beta} \Phi_{3, \beta}\right] \\
\mathcal{L}_{3} u & =\frac{1}{\rho}\left[(\mu+\kappa) \Delta w_{3}+C_{3} \Delta \Phi_{3}+\kappa \varepsilon_{3 \alpha \beta} \Phi_{\beta, \alpha}\right] \\
\mathcal{M}_{\nu} u & =\frac{1}{J}\left[C_{3} \Delta w_{\nu}+\left(C_{1}+C_{2}\right) w_{\rho, \rho \nu}+\gamma \Delta \Phi_{\nu}\right. \\
& \left.+(\alpha+\beta) \Phi_{\rho, \rho \nu}+\kappa \varepsilon_{\nu \rho 3}\left(w_{3, \rho}-v_{\rho}\right)+2\left(C_{3}-C_{2}\right) \varepsilon_{3 \alpha \beta} \Phi_{3, \beta}-2 \kappa \Phi_{\alpha}\right] \\
\mathcal{M}_{3} u & =\frac{1}{J}\left[C_{3} \Delta w_{3}+\gamma \Delta \Phi_{3}+\kappa \varepsilon_{3 \rho \alpha} w_{\alpha, \rho}\right. \\
& \left.+2 \varepsilon_{3 \alpha \beta}\left(C_{3}-C_{2}\right) \Phi_{\beta, \alpha}+C_{2} v_{\alpha, \alpha}-2 \kappa \Phi_{3}\right] \\
\mathcal{H}_{\alpha} u & =\frac{1}{\rho I}\left\{I\left[(\mu+\kappa) \Delta v_{\alpha}+(\lambda+\mu) v_{\rho, \rho \alpha}\right]\right. \\
& \left.-2 h\left[\mu w_{3, \alpha}+C_{2} \Phi_{3, \alpha}+\kappa \varepsilon_{3 \beta \alpha} \Phi_{\beta}+(\mu+\kappa) v_{\alpha}\right]\right\} \\
g_{\alpha} & =f_{3+\alpha}+\frac{1}{\rho} b_{1} T_{1, \alpha}, g_{3}=f_{6}, g_{3+\alpha}=f_{9+\alpha}+\frac{1}{J} b_{2} T_{1, \alpha} \\
g_{6} & =f_{12}, g_{6+\alpha}=f_{14+\alpha}+\frac{1}{\rho I} b_{1} T_{2, \alpha}
\end{aligned}
$$


Let $u=\left(w_{i}, \Phi_{i}, v_{\alpha}\right)$ and $u^{*}=\left(w_{i}^{*}, \Phi_{i}^{*}, v_{\alpha}^{*}\right)$. To study the system (80) we introduce the bilinear form

$$
\Lambda\left(u, u^{*}\right)=\int_{\Sigma} \Pi d a
$$

where

$$
\Pi=2 h\left[\rho w_{j}^{*} \mathcal{L}_{j} u+J \Phi_{j}^{*} \mathcal{M}_{j} u\right]+\rho I v_{\alpha}^{*} \mathcal{H}_{\alpha} u
$$

By using the divergence theorem and the boundary conditions we can see that $\Lambda$ is a bounded bilinear form. In view of (67) we find that this form is coercive. On the basis of Lax-Milgram theorem (see, e.g., [23]) we obtain the existence of solution of the system (80). We conclude that the equation (77) has a solution in $\mathcal{D} . \square$

A direct consequence of the Lumer-Phillips theorem (see [24]) is the following result.

Theorem 6. The operator $\mathcal{A}$ is the generator of a $C^{\infty}$-semigroup of contraction in the Hilbert space $\mathcal{Z}$.

Thus we can state the main result of this section

Theorem 7. Let $\mathcal{B} \in C^{1}\left(\mathbb{R}^{+}, \mathcal{Z}\right) \cap C^{0}\left(\mathbb{R}^{+}, \mathcal{D}\right)$ and $w_{0} \in \mathcal{D}$. Then, there exists a unique solution $\omega \in C^{1}\left(\mathbb{R}^{+}, \mathcal{Z}\right) \cap C^{0}\left(\mathbb{R}^{+}, \mathcal{D}\right)$ to the dynamic problem.

\section{DEFORMATION OF A PLATE WITH A CIRCULAR HOLE}

In this section we study the deformation of an infinite plate with a circular hole, subjected to a constant temperature at the periphery of the hole. The domain $\Sigma$ is defined by $\Sigma=\left\{\left(x_{1}, x_{2}, x_{3}\right): x_{1}^{2}+x_{2}^{2}>b^{2}, x_{3}=0\right\}$ where $b$ is a positive constant. We 
introduce the notation $r=\left(x_{1}^{2}+x_{2}^{2}\right)^{1 / 2}$. We consider the equilibrium theory and assume that

$$
\begin{aligned}
& F_{j}=0 G_{j}=0 H_{\alpha}=0 \quad S_{\alpha}=0 \text { on } \Sigma \\
& \widetilde{\tau}_{k}=0 \widetilde{\mu}_{k}=0 \widetilde{M}_{\alpha}=0 \widetilde{T}_{1}=T^{*} \widetilde{T}_{2}=0 \text { on } r=b
\end{aligned}
$$

where $T^{*}$ is a prescribed constant. In this case the static version of the equations (18) reduces to

$$
\begin{aligned}
& (\mu+\kappa) \Delta w_{\alpha}+(\lambda+\mu) w_{\rho, \rho \alpha}+C_{3} \Delta \Phi_{\alpha}+\left(C_{1}+C_{2}\right) \Phi_{\rho, \rho \alpha}+\kappa \varepsilon_{3 \alpha \beta} \Phi_{3, \beta}=b_{1} T_{1, \alpha} \\
& (\mu+\kappa) \Delta w_{3}+C_{3} \Delta \Phi_{3}+\kappa \varepsilon_{3 \alpha \beta} \Phi_{\beta, \alpha}+\mu v_{\alpha, \alpha}=0 \\
& C_{3} \Delta w_{\alpha}+\left(C_{1}+C_{2}\right) w_{\rho, \rho \alpha}+\gamma \Delta \Phi_{\alpha}+(\alpha+\beta) \Phi_{\rho, \rho \alpha}+\kappa \varepsilon_{3 \alpha \rho}\left(w_{3, \rho}-v_{\rho}\right) \\
& +2\left(C_{3}-C_{2}\right) \varepsilon_{3 \alpha \beta} \Phi_{3, \beta}-2 \kappa \Phi_{\alpha}=b_{2} T_{1, \alpha} \\
& C_{3} \Delta w_{3}+\gamma \Delta \Phi_{3}+\kappa \varepsilon_{3 \rho \alpha} w_{\alpha, \rho}+2 \varepsilon_{3 \alpha \beta}\left(C_{3}-C_{2}\right) \Phi_{\beta, \alpha}+C_{2} v_{\alpha, \alpha}-2 \kappa \Phi_{3}=0 \\
& I\left[(\mu+\kappa) \Delta v_{\alpha}+(\lambda+\mu) v_{\rho, \rho \alpha}\right]-2 h\left[\mu w_{3, \alpha}\right. \\
& \left.+C_{2} \Phi_{3, \alpha}+\kappa \varepsilon_{3 \beta \alpha} \Phi_{\beta}+(\mu+\kappa) v_{\alpha}\right]=I b_{1} T_{2, \alpha} \\
& \Delta T_{1}=0 \Delta T_{2}-\nu^{2} T_{2}=0
\end{aligned}
$$

where $\nu=(2 h / I)^{1 / 2}$. It follows from (82) and (83) that the functions $T_{1}$ and $T_{2}$ are given by

$$
T_{1}=T^{*} T_{2}=0
$$

We seek the solution of the equations (83) in the form

$$
w_{\alpha}=F_{, \alpha} w_{3}=0 \Phi_{\alpha}=G_{, \alpha} \Phi_{3}=0 \quad v_{\alpha}=\varepsilon_{3 \alpha \beta} H_{, \beta}
$$


where $F, G$ and $H$ are unknown function of $r$. It is a simple matter to see that the equations (83) are satisfied if the functions $F, G$ and $H$ satisfy the following equations

$$
\begin{aligned}
& (\lambda+2 \mu+\kappa) \Delta F+\left(C_{1}+C_{2}+C_{3}\right) \Delta G=0 \\
& \left(C_{1}+C_{2}+C_{3}\right) \Delta F+(\alpha+\beta+\gamma)\left(\Delta-p_{1}^{2}\right) G+\kappa H=0 \\
& (\mu+\kappa)\left(\Delta-\nu^{2}\right) H+\kappa \nu^{2} G=0
\end{aligned}
$$

where

$$
p_{1}^{2}=2 \kappa /(\alpha+\beta+\gamma)
$$

The equations (86) implies that the functions $G$ and $H$ satisfy the system

$$
\left(\Delta-p_{2}^{2}\right) G+a_{1} H=0\left(\Delta-\nu^{2}\right) H+\ell_{1} G=0
$$

where we have introduced the notations

$$
\begin{aligned}
& p_{2}^{2}=2 \kappa(\lambda+2 \mu+\kappa) / d a_{1}=\kappa(\lambda+2 \mu+\kappa) / d \\
& d=(\lambda+2 \mu+\kappa)(\alpha+\beta+\gamma)-\left(C_{1}+C_{2}+C_{3}\right)^{2} \ell_{1}=\kappa \nu^{2} /(\mu+\kappa)
\end{aligned}
$$

We consider the representation

$$
G=\left(\Delta-\nu^{2}\right) \Lambda H=-\ell_{1} \Lambda
$$

where $\Lambda$ is a function of class $C^{4}$. Let us denote

$$
D=\Delta \Delta-\left(\nu^{2}+p_{2}^{2}\right) \Delta+p_{2}^{2} \nu^{2}-a_{1} \ell_{1}
$$

The functions $G$ and $H$ given by (88) satisfy the equations (56) if the function $\Lambda$ satisfies the equation

$$
D \Lambda=0
$$


We can prove this assertion by substituting the functions $G$ and $H$ from (88) into system (87). The operator $D$ can be expressed in the form

$$
D=\left(\Delta-k_{1}^{2}\right)\left(\Delta-k_{2}^{2}\right)
$$

where $k_{\alpha}^{2}$ are the roots of the equation

$$
y^{2}-\left(\nu^{2}+p_{2}^{2}\right) y+p_{2}^{2} \nu^{2}-a_{1} \ell_{1}=0
$$

Let assume that $k_{\alpha}$ are distinct positive constants. We can write

$$
\Lambda=A_{1} e_{1}+A_{2} e_{2}
$$

where $A_{\alpha}$ are arbitrary constants and the functions $e_{\beta}$ satisfy the equations

$$
\left(\Delta-k_{\alpha}^{2}\right) e_{\alpha}=0 \quad(\text { no sum } ; \alpha=1,2)
$$

The functions $e_{\alpha}$ that satisfy the equations (91) and vanish at infinity are given by

$$
e_{\alpha}=K_{0}\left(k_{\alpha} r\right) \quad(\alpha=1,2,3)
$$

where $K_{0}$ denotes the modified Bessel function of the third kind and zeroth order. Thus, we find that

$$
\Lambda=\sum_{\alpha=1}^{2} A_{\alpha} K_{0}\left(k_{\alpha} r\right)
$$

In view of (88) and (92) we obtain

$$
G=\sum_{\alpha=1}^{2} b_{1 \alpha} A_{\alpha} K_{0}\left(k_{\alpha} r\right) H=\sum_{\alpha=1}^{2} b_{2 \alpha} A_{\alpha} K_{0}\left(k_{\alpha} r\right)
$$


where

$$
b_{1 \alpha}=k_{\alpha}^{2}-\nu^{2} \quad b_{21}=b_{22}=-\ell_{1}
$$

From (86) we find that the function $F$ has the form

$$
F=-\vartheta G+A_{0}+A_{3} \ln r
$$

where $A_{0}$ and $A_{3}$ are arbitrary constants and

$$
\vartheta=\left(C_{1}+C_{2}+C_{3}\right) /(\lambda+2 \mu+\kappa)
$$

It follows from (16), (17), (84) and (85) that on the boundary $r=b$ we have

$$
\begin{aligned}
& \tau_{\beta \alpha} n_{\beta}=n_{\alpha}\left[\lambda \Delta F+(2 \mu+\kappa) F^{\prime \prime}+C_{1} \Delta G+\left(C_{2}+C_{3}\right) G^{\prime \prime}-b_{1} T^{*}\right] \\
& \mu_{\beta \alpha} n_{\beta}=n_{\alpha}\left[\alpha \Delta G+(\beta+\gamma) G^{\prime \prime}+C_{1} \Delta F+\left(C_{2}+C_{3}\right) F^{\prime \prime}-b_{2} T^{*}\right] \\
& \tau_{\alpha 3} n_{\alpha}=0 \mu_{\alpha 3} n_{\alpha}=0 M_{\beta \alpha} n_{\beta}=\varepsilon_{3 \alpha \beta} n_{\beta} I\left[(\mu+\kappa) H^{\prime \prime}-\mu b^{-1} H^{\prime}\right]
\end{aligned}
$$

where $f^{\prime}=d f / d r$ and $f^{\prime \prime}=d^{2} f / d r^{2}$. In view of (20), (82) and (95) the boundary conditions on the boundary $r=b$ become

$$
\begin{aligned}
& \lambda \Delta F+(2 \mu+\kappa) F^{\prime \prime}+C_{1} \Delta G+\left(C_{2}+C_{3}\right) G^{\prime \prime}=b_{1} T^{*} \\
& \alpha \Delta G+(\beta+\gamma) G^{\prime \prime}+C_{1} \Delta F+\left(C_{2}+C_{3}\right) F^{\prime \prime}=b_{2} T^{*} \\
& b(\mu+\kappa) H^{\prime \prime}-\mu H^{\prime}=0 \text { on } r=b
\end{aligned}
$$

If we use (93) and (94), then the conditions (96) reduce to

$$
\begin{aligned}
& \sum_{\alpha=1}^{2} c_{1 \alpha} A_{\alpha}-(2 \mu+\kappa) b^{-2} A_{3}=b_{1} T^{*} \\
& \sum_{\alpha=1}^{2} c_{2 \alpha} A_{\alpha}-\left(C_{2}+C_{3}\right) b^{-2} A_{3}=b_{2} T^{*} \sum_{\alpha=1}^{2} c_{3 \alpha} A_{\alpha}=0
\end{aligned}
$$


where

$$
\begin{aligned}
c_{1 \alpha} & =\left\{\left[C_{1}+C_{2}+C_{3}-\vartheta(\lambda+2 \mu+\kappa)\right] k_{\alpha}^{2} K_{0}\left(k_{\alpha} b\right)\right. \\
& \left.+\left[C_{2}+C_{3}-\vartheta(2 \mu+\kappa)\right] b^{-1} k_{\alpha} K_{1}\left(k_{\alpha} b\right)\right\} b_{1 \alpha} \\
c_{2 \alpha} & =\left\{\left[\alpha+\beta+\gamma-\vartheta\left(C_{1}+C_{2}+C_{3}\right)\right] k_{\alpha}^{2} K_{0}\left(k_{\alpha} b\right)\right. \\
& \left.+\left[\beta+\gamma-\vartheta\left(C_{2}+C_{3}\right)\right] k_{\alpha} b^{-1} K_{1}\left(k_{\alpha} b\right)\right\} b_{1 \alpha} \\
c_{3 \alpha} & =b k_{\alpha} b_{2 \alpha}\left\{(\mu+\kappa)\left[k_{\alpha} K_{0}\left(k_{\alpha} b\right)+b^{-1} K_{1}\left(k_{\alpha} b\right)\right]+\mu\left(k_{\alpha} b\right)\right\}
\end{aligned}
$$

Here, $K_{1}$ denotes the modified Bessel function of the third kind and order 1. From (97) we determine the constants $A_{s}$. The solution of the problem is given by (85), (93) and (94). The behaviour of solution at infinity is the same as in the classical theory. In contrast with the theory of achiral plates, a uniform temperature field acting on the boundary of the hole produces a bending effect.

\section{References}

1. R. Lakes, Elastic and Viscoelastic Behavior of Chiral Materials, Int. J. Mech. Sci., vol. 43 , pp. 1579-1589, 2001.

2. R.S. Lakes and R.L. Benedict, Noncentrosymmetry in Micropolar Elasticity, Int. J. Engng. Sci., vol. 29, pp. 1161-1167, 1982.

3. R.S. Lakes, H.S. Yoon and J.L. Katz, Slow Compressional Wave Propagation in Wet Human and Bovine Cortical Bone, Science, vol. 200, pp. 513-515, 1983. 
4. H.C. Park and R.S. Lakes, Cosserat Micromechanics of Human Bone: Strain Redistribution by a Hydration-Sensitive Constituent, J. Biomechanics, vol. 19, pp. 385-397, 1986.

5. R.S. Lakes, Foam Structures with a Negative Poissons Ratio, Science, vol. 235, pp. 1038-1040, 1987.

6. D. Prall and R.S. Lakes, Properties of a Chiral Honeycomb with a Poisson's Ratio- 1, Int. J. Mech. Sci., vol. 39, pp. 305-314, 1997.

7. T.J. Healey, Material Symmetry and Chirality in Nonlinearly Elastic Rods, Math. Mech. Solids, vol. 7, pp. 405-420, 2002.

8. D. Natroshvili, L. Giorgashvili and I.G. Stratis, Representation Formulae of General Solutions in the Theory of Hemitropic Elasticity, Q.J. Mech. Appl. Math., vol. 59, pp. 451-474, 2006.

9. A. Spadoni and M. Ruzzene, Elasto-static micropolar behaviour of a chiral auxetic lattice, J. Mech. Phys. Solids, vol. 60, pp. 156-171, 2012.

10. K. Chandraseker, S. Mukherjee, J.T. Paci and G.C. Schatz, An Atomistic-Continuum Cosserat Rod Model of Carbon Nanotubes, J. Mech. Phys. Solids, vol. 57, pp. 932-958, 2009.

11. W. Nowacki, Theory of Asymmetric Elasticity, PWN, Warszawa, 1981. 
12. A.C. Eringen, Microcontinuum Field Theories, I: Foundations and Solids, Springer, New York, Berlin, 1999.

13. J. Dyszlewicz, Micropolar Theory of Elasticity, Springer, Berlin, New York, 2004. Press, London, New York, Boca Raton, 2009.

14. J. Altenbach, H. Altenbach and V.A. Eremeyev, On generalized Cosserat type theories of plates and shells: a short review and bibliography, Arch. Appl. Mech. vol. 80 , pp. 73-92,2010.

15. A.C. Eringen, Theory of micropolar plates, Jour. Appl. Math. Phys. ZAMP, vol. 18, pp. 12-13, 1967.

16. W. Nowacki, Thermoelasticity, Pergamon Press, Oxford, London, New York, Paris, 1962.

17. E. Inan, Coupled theory of thermoelastic plates, Acta Mechanica, vol. 14, pp. 1-29, 1972.

18. L. Brun, Methodes energetiques dans les systemes evolutifs lineaires. J. Mécanique, vol. 8, pp. 125-192, 1969.

19. D. Ieşan, On some theorems in thermoelastodynamics, Rev. Roum. Sci. Techn. Mec. Appl., vol. 34, pp. 101-111, 1989.

20. D. Ieşan, Thermoelastic Models of Continua, Kluwer Acad. Publ., Dordrecht, 2004 . 
21. N.S. Wilkes, Continuous dependence and instability in linear thermoelasticity, SIAM J. Math. Anal., vol. 11, pp. 292-299, 1980.

22. J.A. Goldstein, Semigroup of Linear Operators and Applications, Oxford University Press, Oxford, 1985.

23. D. Gilbarg and N.S. Trudinger, Elliptic Partial Differential Equations of Second Order, Springer Verlag, Berlin, 1983.

24. Z. Liu and S. Zheng, Semigroup Associated with Dissipative Systems, CRC Research Notes in Mathematics vol. 398, Chapmann \& Hall, Boca Raton, 1999. 